\title{
HAIR LOSS ASSOCIATED WITH ESCITALOPRAM: DO SSRIS AFFECT MELATONIN AT THE HAIR FOLLICLE?
}

\author{
İrem Yazıcı Karabulut, Hasan Gokcay \& Hasan Belli \\ Department of Psychiatry, University of Health Sciences, Bagcilar Training and Research Hospital, Istanbul, Turkey \\ received: 16.1.2021; \\ revised: 25.2.2021; \\ accepted: 2.3 .2021
}

\section{INTRODUCTION}

Antidepressant drugs are among the most prescribed drugs worldwide because of their high reliability and low side effect profiles in the treatment. Selective serotonin reuptake inhibitors (SSRIs) can be used in the treatment of many psychiatric disorders such as major depressive disorder, generalized anxiety disorder, panic disorder (Bahrick \& Audrey 2008). At the same time, it is frequently prescribed in cases of dermatological diseases such as atopic dermatitis, psoriasis, alopecia areata accompanied by psychiatric diseases or psychiatric symptoms (Gupta \& Gupta 1996). As far as we know, dermatological patients have a higher prevalence of psychiatric comorbidity in the general population. It is estimated that $25-30 \%$ of patients have certain mental disorders or emotional problems that may represent the cause, predisposition, or severity of the skin condition (Gee et al. 2013). On the other hand, skin disease can also bring some emotional problems. In such cases, antidepressant combination of dermatological treatment may be considered. SSRIs are commonly used antidepressants that are generally safer than alternatives. Several skin reactions have been reported as side effects of these drugs, including bruising, pruritus, urticaria, angioedema, erythema multiforme, Stevens-Johnson syndrome, toxic epidermal necrolysis, erythema nodosum, alopecia, hypertrichosis, leukocytoclastic vasculitis, and an acneiform eruption (Krasowska et al. 2007). As far as we know, hair loss caused by escitalopram is a rare side effect and there are very few cases in the literature on this subject. Our study aimed to the contribute the literature and physicians in clinical practice.

\section{CASE}

A 22-year-old university student male patient was admitted to our psychiatry outpatient clinic with restlessness, discomfort, anhedonia, insomnia, fatigue, malaise, and irritability, reluctance and depressed mood. He lost appetite and lost 8 kilograms in three months. In the psychiatric examination, the patient was conscious, cooperative, full oriented with restlessness, Burns depression checklist total score was 71 . He had no other illness other than psychiatric complaints and did not use any medication.
Its diagnosis was considered as major depressive disorder according to the Diagnostic and Statistical Manual of Mental Disorders, $5^{\text {th }}$ edition . Escitalopram $10 \mathrm{mg}$ treatment was started. After one month of treatment, the total score of Burns depression checklist was 41 . Meanwhile, bupropion $150 \mathrm{mg}$ was added in the second month of the treatment, as the patient's complaints of cognitive impairment, inattention, inability to gather the mind, and smoking exceeding one package per day continued. One month after starting bupropion. The patient complained of active hair loss and the dermatology was consulted; complete blood count, anemic profile (serum ferritin, iron, iron- binding capacity, serum folate, and serum vitamin B12), gonadal steroid hormones (total testosterone, DHEA-S), thyroid levels (T3, T4, $\mathrm{TSH}$ ), liver and kidney functions, serum electrolytes (Na, $\mathrm{K}, \mathrm{Ca}, \mathrm{Cl}$ and $\mathrm{Mg}$ ), copper and zinc tests were performed. No pathology was found in blood analysis and examination. Bupropion was stopped due to suspicion of the side effects and escitalopram $10 \mathrm{mg}$ was continued.

Antidepressant treatment continued with escitalopram $10 \mathrm{mg}$ for 2 more months. The patient's depressive complaints decreased, cognitive functions improved, akathisia disappeared. However, the hair loss continued. Naranjo Adverse Drug Reaction Probability Scale score was 6 points (a probable relationship). The patient could not tolerate hair loss and switch from escitalopram to vortioxetine $10 \mathrm{mg} / \mathrm{day}$. The hair loss stopped in the first month of the switch, then it did not recur and Burns depression checklist total score was 17 . The patient's treatment continued with vortioxetine $10 \mathrm{mg} /$ day.

\section{DISCUSSION}

Hair loss has previously been reported with escitalopram and other serotonin reuptake inhibitors and serotonin-norepinephrine reuptake inhibitors (SNRIs) such as venlafaxine (Etminan et al. 2018). Studies have found that all other antidepressants have a lower risk of hair loss compared to Bupropion. (Etminan et al. 2018). The mechanism of escitalopram-associated hair loss has not been completely elucidated.

Citalopram is a racemic serotonin inhibitor, containing the $\mathrm{r}$ and s-enantiomer. The s-enantiomer is the active component and is marketed as escitalopram. The active molecule of escitalopram in plasma is non- 
metabolized escitalopram. The main metabolite is $\mathrm{S}$ demethylcitalopram (S-DCT) and is present at approximately one-third of escitalopram. S-DCT is a negligible inhibitor of serotonin reuptake and has no significant role in therapeutic activity (Rao \& Niranjan 2007). Escitalopram is considered an SSRI with the least CYPrelated drug, also with low protein-binding $(56 \%)$ ratio is an advantage of low interaction with highly proteinbound drugs. It suggests that escitalopram is unlikely to cause clinically significant drug-drug interactions. Therefore, escitalopram has an advantageous pharmacokinetic profile that provides clinical benefits to a wide range of patients (Rao \& Niranjan 2007).

The effects of serotonin on skin and hair are thought to be mediated by melatonin. One of the main sources of melatonin in the body is serotonin. Human scalp hair follicles are both a target and one of the extrapineal synthesis sites of melatonin (Kobayashi et al. 2005). Roles of melatonin in the skin are related to epidermal metabolic regulation, photoprotection, pigmentation, protection from oxidative stress, DNA repair, anticancer activity, wound healing, and hair follicles. Melatonin acts on the skin through MT1, MT2, and nuclear ROR $\alpha$ receptors. MT1 was detected in the hair follicle, MT2 has detected blood vessels of human skin (Slominski et al. 2018). In vitro melatonin has been shown to increase animal hair follicles, therefore, it is thought to benefit hair loss by increasing anagen hair follicles in women (Kobayashi et al. 2005).

Melatonin plays a role in the hair growth cycle (Slominski et al. 2005). Therefore SSRIs can cause side effects related to hair and skin, they are thought to damage the telogen phase of hair production, change the structure and color of the hair (Mercke et al. 2000). However, this aspect of the mechanism of action is not entirely explained. Considering that these drugs are used frequently, cases of alopecia may not be noticed in patients with depression because they do not mention hair loss as a result of self- neglect, or present hair loss may not be associated with the medication.

We still do not know enough about the SSRI mechanism, one of the commonly used drugs, but we have certainly had a lot to learn from our patients. With the recognition and sharing of rare side effects, it will inspire research on this subject and lead to the elucidation of mechanisms. We hope the case study will be useful to clinicians facing hair loss cases with escitalopram.
Acknowledgments: None.

Conflict of interest: None to declare.

Contribution of individual authors:

İrem Yazıcı Karabulut: data collection.

Hasan Gokcay \& Hasan Belli: first draft.

\section{References}

1. Bahrick, Audrey S: Persistence of Sexual Dysfunction Side Effects after Discontinuation of Antidepressant Medications: Emerging Evidence. The Open Psychology Journal 2008; $1: 42-50$

2. Etminan, Mahyar, Mohit Sodhi, Ric M. Procyshyn, Michael Guo et al.: Risk of Hair Loss with Different Antidepressants: A Comparative Retrospective Cohort Study. International Clinical Psychopharmacology 2008; 33:44-48

3. Gee SN, Zakhary L, Keuthen N, Kroshinsky D \& Boer Kimball A: A Survey Assessment of the Recognition and Treatment of Psychocutaneous Disorders in the Outpatient Dermatology Setting: How Prepared Are We? Journal of the American Academy of Dermatology 2013; 68:47-52

4. Gupta MA \& Gupta AK: Psychodermatology: An Update. Journal of the American Academy of Dermatology 1996; 34:1030-46

5. Kobayashi H, Kromminga A, Dunlop TW, Tychsen B, Conrad F et al.: A Role of Melatonin in Neuroectodermalmesodermal Interactions: The Hair Follicle Synthesizes Melatonin and Expresses Functional Melatonin Receptors. The FASEB Journal 2005; 19:1710-12

6. Krasowska D, Szymanek M, Schwartz RA, Myśliński W: Cutaneous Effects of the Most Commonly Used Antidepressant Medication, the Selective Serotonin Reuptake Inhibitors. Journal of the American Academy of Dermatology 2007; 56:848-53

7. Mercke Y, Sheng H, Khan T, Lippmann S: Hair Loss in Psychopharmacology Annals of Clinical Psychiatry 2000; 12:35-42

8. Rao N: The Clinical Pharmacokinetics of Escitalopram. Clinical Pharmacokinetics 2007; 46:281-90

9. Slominski AT, Hardeland R, Zmijewski MA, Slominski RM, Reiter RJ et al.: Melatonin: A Cutaneous Perspective on Its Production, Metabolism, and Functions. Journal of Investigative Dermatology 2008; 138:490-99

10. Slominski A, Wortsman J, Tobin DJ: The Cutaneous Serotoninergic/Melatoninergic System: Securing a Place under the Sun. The FASEB Journal 2005; 19:176-94

\section{Correspondence:}

Hasan Gökçay, MD

Department of Psychiatry, University of Health Sciences, Bagcilar Training and Research Hospital

34200, Istanbul, Turkey

E-mail: hasangkcy@yahoo.com 